Exper i ment al I nvest i gat $\mathrm{i}$ on of an Adapt i vel y Tuned Dynamic Absor ber I ncor por at i ng Magnet or heol ogi cal El ast omer wi th Sel f-Sensi ng Property

\begin{tabular}{|l|l|}
\hline 著者 & $\begin{array}{l}\text { Konat suzaki Toshi hi ko, I noue Toshi o, I wat a } \\
\text { Yoshi o }\end{array}$ \\
\hline $\begin{array}{l}\text { j our nal or } \\
\text { publ i cat i on t i t l e }\end{array}$ & Exper i ment al Nechani cs \\
\hline vol une & 56 \\
\hline number & 5 \\
\hline page range & $871-880$ \\
\hline year & $2016-06-01$ \\
\hline URL & ht t p: //hdl . handl e. net /2297/44880 \\
\hline
\end{tabular}




\title{
Experimental Investigation of an Adaptively Tuned Dynamic Absorber Incorporating Magnetorheological Elastomer with Self-sensing Property
}

\author{
Toshihiko Komatsuzaki $^{1, *}$, Toshio Inoue ${ }^{2}$, Yoshio Iwata ${ }^{1}$ \\ ${ }^{1}$ Kanazawa University, Institute of Science and Engineering, Kakuma-machi, Kanazawa, Ishikawa \\ 920-1192 JAPAN \\ E-mail:iwata@se.kanazawa-u.ac.jp \\ ${ }^{2}$ Honda R\&D Co., Ltd., Automobile R\&D Center \\ 4630 Shimotakanezawa, Haga-machi, Haga-gun, Tochigi, 321-3393 JAPAN \\ E-mail:Toshio_Inoue@n.t.rd.honda.co.jp \\ * Corresponding author. Tel.: +81 76234 4673; fax: +81 762344676 \\ E-mail:toshi@se.kanazawa-u.ac.jp
}

\begin{abstract}
The magnetorheological elastomer (MRE) is known to belong to a class of smart materials whose elastic properties can be varied by an externally applied magnetic field. In addition to the property of the field-dependent stiffness change of the MRE, the electrical resistance of the composite is also changed by the induced strain, thereby providing a new selfsensing feature. In the present study, a novel, dynamic vibration absorber is developed using an MRE with a self-sensing function and adaptability. The natural frequency of the absorber is instantaneously tuned to a dominant frequency extracted from the strain signal of MRE. The damping performance test shows that the vibration of a system with one degree-of-freedom that is exposed to a nonstationary disturbance can be adequately reduced by the proposed frequency-tunable dynamic absorber without the use of external sensors.
\end{abstract}

\section{Keywords}

Magnetorheological elastomer, Dynamic absorber, Variable stiffness, Electric conductivity, Frequency tunability, Self-sensing property 


\section{Acknowledgements}

The work was supported by the Japan Society for the Promotion of Science (JSPS)

KAKENHI, Grant-in-Aid for Scientific Research (B), Grant Number 15 H03936. 


\section{Introduction}

A passive-type dynamic vibration absorber (DVA) is especially effective when the excitation frequency is close to the natural frequency of the structure since the natural frequency of the DVA is usually tuned to a particular excitation frequency [1]. Fixing the physical properties of the DVA limits the application to a narrowband, harmonically excited vibration problem. Any mechanical component types that can modulate their stiffness provide adaptability to the DVA against nonstationary disturbances [2-5] but the implementation of such adaptability might be complex.

The magnetorheological elastomer (MRE) is a type of a smart material whose viscoelastic property can be varied according to an externally applied magnetic field. A number of studies have been reported on the fundamental features of MREs [6-8]. These research studies share common findings whereby the baseline stiffness of the composite was kept low, and the amount of the magnetic filler particles was near the critical concentration, even for the largest changes of the material properties. Attempts have also been expanded on enhancing the viscoelastic property change for the anisotropic (or structured) MREs. Stepanov et al. [9] compared the viscoelastic properties of isotropic and structured soft MREs using static and dynamic shearing tests that changed the size distribution of the magnetic particles. They have found that the largest modulus increase against the applied magnetic field was observed for a structured sample with wider particle size distribution. While all these investigations mainly focused on the evaluation of the viscoelastic property along the shear direction, the material property of MRE in tensile, compression, and squeeze modes, were also studied $[9,10]$. Others have suggested the theoretical models explaining the mechanism of the MR effect and numerically predicted the mechanical response of MREs [11-15]. 
Another prior research study refers to the application of MREs to semi-active vibration control devices, such as the frequency-tunable DVAs. Deng and Gong [16] have proposed a tunable absorber using an MRE for which a natural frequency shift of $155 \%$ was obtained when a magnetic field of $1 \mathrm{~T}$ was applied that consequently damped the beam vibration effectively. Learner and Cunefare [17] have studied MRE-based vibration absorbers in which MREs were deformed under three different configurations: under shear, squeeze, and compression modes. They have found that MREs with an iron concentration of $35 \%$ by volume yielded the largest natural frequency shift of 507\% when incorporated in a squeezemode absorber. Popp et al. [18] also analyzed the mechanical performance of MRE-based vibration absorbers which operate in shear and squeeze modes. They have found the different working frequency ranges for both modes.

Regardless of the choice of a real-time controller design, the state of the target structure should always be monitored against unknown disturbances so that an appropriate control signal for active/semi-active devices can be supplied by the controller [19]. Any type of an external sensor is usually introduced to monitor the dynamic response of the structures, while leaving cost and maintenance problems for real applications. In addition to the magnetic-field-dependent stiffness change properties of an MRE, the electrical resistance of the material is also changed owing to the induced strain within the elastomer, thereby providing a new self-sensing feature. Wang et al. [20] investigated the sensing behavior of MREs at different weight fractions of iron particles under uniaxial compression loading by an impedance spectroscopic technique. They have found that the electrical resistance of the samples changes depended on both the magnitude of the mechanical loading and the strength of the magnetic field. In several other studies, it has been shown that a wider electrical resistance change of the composite can be obtained, and the sensitivity to the 
applied external force can be improved by the addition of graphite particles [21-23]. The change of the material conductivity for both the applied loading and the magnetic field could be explained by the change in the distance between particles.

The force/strain sensing property mentioned above can be used for the operation of the semi-active vibration devices that have self-sensing features. However, a highly reproducible sensing characteristic might be obtained for thin samples, whose thicknesses are limited to values within a few millimeters. One way of avoiding such limitation is to mold the sensing and the variable-stiffness parts separately, and then bond them together. In this case, the filler is not necessarily a mixture of magnetic and nonmagnetic particles for the expression of electrical conductivity. Considering that the magnetic field temporally varies in real-time control situations, it is preferable to use nonmagnetic particles for the sensing portion of the MRE, in such a way that the deformation response is obtained irrespective of the magnetic field. To the best of our knowledge, the use of the sensor function in parallel with the stiffness controllable feature has yet to be investigated.

In this paper, the stiffness controllable magnetorheological elastomer is used in the dynamic absorber whose natural frequency can be tuned by an external magnetic field. The present investigation focuses on the development of a novel DVA with a self-sensing function and adaptability. The newly developed DVA has a distinctive self-contained feature, whose natural frequency can be tuned automatically according to the self-detected strain signal by the MRE incorporated in the system without the use of an external displacement sensor. By obtaining the strain signal from a change in the electrical resistance of the elastomer, the natural frequency of the absorber is instantaneously tuned, and the real-time vibration control performance of the absorber for a system with one degree-of-freedom is evaluated. 


\section{Viscoelastic property of the magnetorheological elastomer}

The elastomeric composite incorporating ferrous particles, known as the magnetorheological elastomer (MRE), has been developed with the aim to overcome the problems associated with the magnetorheological fluid (MRF), such as the sedimentation and the aggregation of particles [24]. Under the presence of a magnetic field, magnetically polarized particles form chain-like structures according to the attractive forces amongst each other that produce changes in the apparent viscoelastic property of the elastomer.

The fabricated MRE samples in the present study are composed of carbonyl iron powder with an approximate diameter of $10 \mu \mathrm{m}$ dispersed within the room temperature vulcanizing (RTV) rubber matrix. Under the presence of an external magnetic field, anisotropic elastomer samples are formed in square cuboids with a size of $25 \mathrm{~mm}$ and a thickness of $10 \mathrm{~mm}$, and with different iron volume contents ranging from 30 to $50 \%$.

The viscoelastic properties of the fabricated MREs are investigated by measuring their dynamic response using the experimental setup shown in Fig. 1. The upper part of the electromagnet, and two MREs placed between the upper and the lower parts of the electromagnet are assumed to respectively emulate a mass and two springs, and they constitute a one degree-of-freedom vibration system. While exciting the system horizontally by an exciter, the base and mass accelerations are measured and processed in the form of a transfer function by a Fast Fourier Transform (FFT) spectrum analyzer. The frequency response of the system is measured through a white-noise random excitation at the cutoff frequency of $100 \mathrm{~Hz}$, and the root mean square (RMS) acceleration level is $0.5 \mathrm{G}$. The applied electric current is varied from 0 to $6 \mathrm{~A}$.

The frequency dependent complex spring constant $k^{*}$ is defined as follows [25]: 


$$
k^{*}(\omega)=k_{0} \cdot v(\omega)\{1+j \eta(\omega)\}
$$

In Eq. (1), $k_{0}$ signifies the reference spring constant calculated by the natural frequency $\omega_{0}$ of the system when no magnetic field is applied, $\omega$ the frequency of excitation, $v(\omega)$ is the frequency-dependent normalized spring constant, $\eta(\omega)$ is the loss factor and $j$ is a complex number, $j=\sqrt{-1}$. Using symbols $v(\omega)$ and $\eta(\omega)$, the acceleration transfer function between the base and the mass, is derived according to the following equation:

$$
G(j \omega)=\frac{v(\omega)+j \eta(\omega) v(\omega)}{\left\{v(\omega)-\lambda^{2}\right\}+j \eta(\omega) v(\omega)}
$$

In Eq. (2), $\lambda=\omega / \omega_{0}$. By separating the real and the imaginary parts of the transfer function as $G(j \omega)=G_{R}+j G_{I}, v(\omega)$ and $\eta(\omega)$ can be expressed using $G_{R}$ and $G_{I}$, as

$$
\begin{aligned}
& v(\omega)=\frac{G_{R}\left(G_{R}-1\right)+G_{I}^{2}}{\left(G_{R}-1\right)^{2}+G_{I}^{2}} \lambda^{2} \\
& \eta(\omega)=\sqrt{-1+\frac{\left(2 G_{R}-1\right) \lambda^{2}}{\left(G_{R}-1\right) v(\omega)}-\frac{G_{R} \lambda^{4}}{\left(G_{R}-1\right) \nu(\omega)^{2}}}
\end{aligned}
$$

The preliminary calculations of $v(\omega)$ and $\eta(\omega)$ as functions of $\omega$ have shown that both properties are dependent on frequency. Although setting these parameters constant might be efficient for analyzing the engineering systems, this approach violates the causality principle [26]. The transient response of the real system cannot be represented properly using such a simplified mathematical model. The choice of the fractional derivative model in time domain is thought to be an appropriate approach for modeling viscoelastic behavior of the MRE [27]. 
Despite the problem mentioned above, the stiffness magnification and the loss factor are averaged over the frequency range to illustrate the dependence of these properties on the magnetic field. These values are shown in Table 1 for MREs synthesized with different iron proportions. The nominal value of the spring constant in the absence of a magnetic

field is $15.9 \mathrm{~N} \mathrm{~mm}^{-1}$ for an MRE of $30 \%$ iron volume content (vol\%), $24.6 \mathrm{~N} \mathrm{~mm}^{-1}$ for a content of $40 \mathrm{vol} \%$, and $58.2 \mathrm{~N} \mathrm{~mm}^{-1}$ for a content of $50 \mathrm{vol} \%$. Based on the nominal values and the sample dimension, the identified shear storage moduli for each MRE are plotted in Fig. 2. The stiffness has the lowest value when no magnetic field is applied, and it becomes larger in value according to the strength of the field owing to the increase in interparticle forces within the elastomeric matrix. In every sample, the modulus tends to saturate as the field increases. In terms of a stiffness magnification based on the nominal value, the maximum variation is observed for the sample with a 40 vol $\%$ iron content. The higher volume content of iron particles leads to the expression of the stronger interparticle forces, whereas the base hardness of the elastomer itself also becomes high. Hence, the largest magnification of stiffness is provided by a certain optimum composition between the elastomeric matrix and the iron particles. On the other hand, the change of the loss factor seems to be small enough in comparison to the change of the stiffness, irrespective of the presence of the magnetic field, and whose value becomes approximately equal to 0.4 in all cases.

\section{An MRE-based variable-stiffness dynamic absorber with a sensor function}

\subsection{Frequency-shift property of the dynamic absorber}

A frequency-tunable DVA is further developed using the aforementioned MRE. A schematic and a photograph of the dynamic absorber that incorporate the MRE as a variable 
stiffness element are shown in Fig. 3. The outer frame is made of steel and constitutes a closed magnetic path. A magnetic coil located at the center works as the mass $(370 \mathrm{~g})$ of the absorber that also generates the magnetic flux. Two MREs with thicknesses of $10 \mathrm{~mm}$ and diameters of $20 \mathrm{~mm}$ are mounted on the upper and lower parts of the coil so that the stiffness will change owing to the magnetic flux penetration. A wire with $1 \mathrm{~mm}$ in diameter is used to wind the coil with five hundred and forty turns.

The ability of the proposed absorber to change both the natural frequency and the

damping ratio against the applied magnetic field was evaluated. The impulse response of the absorber was measured in the form of a time history at each applied current that varied from 0 to $5 \mathrm{~A}$, and the natural frequency and the damping ratio were then calculated. The magnetic flux density increased almost linearly against the applied electric current, and the value was $20 \mathrm{mT}$ for the current of $1 \mathrm{~A}$.

The measurement results of the fundamental properties are summarized in Fig. 4 and Table 2, for an absorber using MREs with a 40 vol\% iron content. The baseline frequency of $25.8 \mathrm{~Hz}$ in the absence of a magnetic field is extended by a factor of 1.45 when a $5 \mathrm{~A}$ current is applied. The observed damping ratio is thought to have an average constant value of 0.12 .

\subsection{Electrical resistance property of a graphite-based elastomer}

In addition to the field-dependent stiffness change property of the MRE, the electrical resistance of the material is also changed according to the induced strain within the elastomer [20-23]. Such a conductive property can be realized by the higher iron powder incorporation within the elastomeric matrix as well as the proper arrangement of the electrically conductive particles into the deforming direction of the host elastomer. 
However, the inclusion of too much iron powder into the entire composite will affect the stiffness change properties of the MRE. Additionally, highly reproducible sensing characteristics might be obtained for thin samples, whose thicknesses are limited within a few millimeters. In the present investigation, a small piece of a commercially available conductive elastomer, as shown in Fig. 5(a), is introduced in order to mimic the sensor portion within a host MRE [28]. The silicone-based conductive elastomer is molded in a cuboid with dimensions of $3 \mathrm{~mm}$ square and a thickness of $1 \mathrm{~mm}$, where the nonmagnetic graphite particles are dispersed. It is embedded into the host MRE as shown in Fig. 5(b). We found that embedding a conductive elastomer into the host MRE has little influence on the original stiffness variation characteristic of the MRE.

Based on the use of the nonmagnetic particles for the sensing portion of MRE, the deformation response is expected, which is dependent only on the external force, irrespective of the magnetic field.

The embedded sensing portion is connected to a resistance voltage divider circuit through the electrodes for a strain-to-voltage conversion. The circuit is schematically shown in Fig. 6 . In the figure, $V_{\text {in }}$ signifies the input voltage, $R_{\text {MRE }}$ the variable resistor corresponding to the sensing portion of MRE, and $R_{\mathrm{M}}$ the measuring resistor, which is chosen to modulate the desired sensitivity range. The sensor output $V_{\text {out }}$ is further obtained by the following equation.

$$
V_{\text {out }}=\frac{R_{\mathrm{M}} V_{\text {in }}}{\left(R_{\mathrm{MRE}}+R_{\mathrm{M}}\right)}
$$

In order to identify the strain-to-voltage conversion that is characteristic of the sensing elastomer, the output voltage associated with the change of the electrical resistance is 
evaluated by both compressive and shear loading experiments. The sensing elastomer alone was statically loaded up to $3.5 \mathrm{~N}$ for compression, whereas it was sheared into the transverse direction until the load reached $1 \mathrm{~N}$. The variation characteristics of the electrical resistance $R_{\mathrm{MRE}}$ and the output voltage $V_{\text {out }}$ are shown in Fig. 7 for respective loading directions. In these experiments, the measuring resistor $R_{\mathrm{M}}$ was set to $12 \Omega$, and the input direct current (DC) voltage $V_{\text {in }}$ was $5 \mathrm{~V}$. The nonlinear relationship between the applied load and the electrical resistance can be seen in both cases. Specifically, the resistance decreases rapidly against loads below $1 \mathrm{~N}$, and it then continues to decrease gradually, as shown in Fig. 7(a). Although the delamination of electrodes might occur at larger loads, the same can be said to hold true for the shear property. In each case, the sensor output voltage increases monotonically from 0 to $1 \mathrm{~V}$ against varying loads that range from 0 to $1 \mathrm{~N}$. Therefore, we can capture the deformation of the elastomer with high sensitivity that is induced by the external disturbances.

Fig. 8 shows examples of the waveform obtained by the embedded sensing elastomer within the DVA for sinusoidal excitations at frequencies of 20,30, and $40 \mathrm{~Hz}$. In the figure, the displacement of the absorber mass $x_{2}$ is simultaneously shown. The electrical resistance of the elastomer changes symmetrically in both shearing directions. It decreases from the neutral state as the deformation increases, irrespective of the deforming directions. It follows that under the sinusoidal disturbance with the constant positive DC input applied to the circuit as $V_{\text {in }}$, the negative part of the disturbance signal appears to be a positively inverted output signal. Hence, the fundamental frequency becomes equal to twice the value of the disturbance signal. In Fig. 8, the signals are put through a high-pass digital filter in order to cut the DC component. By applying the DC blocking filter to the signal, the DC 
component vanishes, but the waveform is distorted and becomes non-symmetrical, due to the presence of the higher order harmonics. Although the preloading of the sensor when it is embedded might also affect the wave distortion, the effect seems to be small.

Despite the distortion of the waveform, the detected signals are periodic, and their frequencies properly correspond to those of the disturbance signals. However, the magnitude of the output signal is not always consistent with the true response amplitude. Fortunately, proving only the instantaneous disturbance frequency information is enough for the tuning of the proposed absorber, regardless of the waveform distortion, only if the detected signal is periodic.

An attempt was made in order to obtain asymmetric output characteristic for the MRE. A biased electrical resistance was provided by a certain amount of preloading when embedding the sensing elastomer into the host MRE. Under the preloaded condition, the elastomer elicited responses whose fundamental frequency corresponds to the value of the disturbance signal as shown in Fig. 9. The frequency responses of the DVA and the output signal are also shown in Fig. 10. In Fig. 10(a), the response is expressed as the transfer function between measured accelerations of the excitation and the absorber mass, and also in Fig. 10(b), the transfer function between the sensor output voltage and the excitation acceleration is shown. The biased MRE exhibits output characteristics similar to an accelerometer in both phase and amplitude. These results show the possibility to use the MRE as a vibration sensor, however, it was found difficult to constantly produce the intended bias resistance in the present DVA configuration. Therefore, the non-biased MRE was introduced in the following vibration control investigations. 


\subsection{Experimental setup and a controller design for a dynamic absorber tuned in real time}

Based on the measured results of the fundamental property of the absorber with variable stiffness, the damping performance is experimentally evaluated for the vibration control of a one-degree-of-freedom (DOF) system, as schematically shown in Fig. 11(a). A photograph of the experimental apparatus is also shown in Fig. 11(b). In the experiment, the absorber incorporating an MRE with a 40 vol\% iron content is adopted. The ratio between the primary and the damper masses is chosen to be 0.25 . The damping ratio of the primary system alone is also found to be 0.01 . The transient response of the system is measured when the entire structure is excited by sweeping the input at the shaking table with a sine wave whose frequency varies from 5 to $55 \mathrm{~Hz}$ at the speed of $2 \mathrm{~Hz} \mathrm{~s}^{-1}$.

The natural frequency of the adjacent system is tuned adaptively to match the frequency of a harmonic external force within the variable range, whereas the damper property is fixed respectively for the lower and upper outer bounds at the minimum or the maximum stiffness value. The switching rule for the stiffness value $k_{\mathrm{MRE}}$ is defined as follows.

$$
k_{\mathrm{MRE}}= \begin{cases}k_{\min } & f<f_{\min } \\ k_{\mathrm{var}} & f_{\min } \leq f \leq f_{\max } \\ k_{\max } & f_{\max }<f\end{cases}
$$

In Eq. (5), $k_{\min }$ and $k_{\max }$ signify the minimum and the maximum stiffness values of an MRE, $f_{\min }$ and $f_{\max }$ the lower and the upper bounds of tunable frequency range, and $f$ the detected disturbance frequency. 


\subsection{Time delay characteristic of the absorber}

The tunable performance of the DVA is strongly related to the transient response property of the absorber. Since the dynamic absorber is intrinsically a passive type device, the transient response is determined by the dynamic property of the device itself: e.g. the natural frequency and the damping ratio. The total time required for the device to be stabilized against the disturbance change is thought to be the sum of the signal processing time required for the electrical devices, and the physically determined transient response time of the absorber.

The time history of the absorber displacement is shown in Fig. 12 for the $20 \mathrm{~Hz}$ sinusoidal excitation. In the figure, two curves are compared. The dotted line shows the response when the stiffness of the absorber is at the lowest value, and the solid curve shows the response when the stiffness is switched corresponding to the applied current of $5 \mathrm{~A}$ at approximately $0.2 \mathrm{~s}$. In accordance with the change of the stiffness, the response amplitude is changed. The system in overall is thought to have the time delay of $0.1 \mathrm{~s}$ until it is stabilized.

Now we estimate the time delay of the present dynamic absorber. The damped free vibration response of a one degree-of-freedom system can be written in general form as follows.

$$
x=e^{-\zeta \omega_{n} t}\left(C \cos \omega_{d} t+D \sin \omega_{d} t\right)
$$

where $\omega_{n}=\sqrt{k / m}, \quad \zeta=c / 2 \sqrt{m k}$, and $\omega_{d}=\omega_{n} \sqrt{1-\zeta^{2}}$. In Eq. (6), $x$ represents the displacement of the system, $\omega_{n}$ the undamped natural frequency, $\zeta$ the damping ratio, and $\omega_{d}$ the damped natural frequency. $C$ and $D$ are coefficients which are determined by the initial condition. Using the natural frequency and the damping ratio values of the absorber, 
$f_{n}=25.8 \mathrm{~Hz}$, and $\zeta=0.12$, we can calculate the time constant $\tau$ of the damped free vibration system as follows.

$$
\tau=\frac{1}{\zeta \omega_{n}}=\frac{1}{0.12 \times(2 \times \pi \times 25.8)}=0.05 \mathrm{~s}
$$

Additionally, it is also known that the regulated power supply has the time constant of 0.05 sec. Therefore, the device has the total time constant of approximately $0.1 \mathrm{~s}$, which almost agrees with the measured time delay in the stiffness switching experiment. The detuning of the absorber occurs at every moment, which might affect the system stability when the disturbance frequency changes quickly.

\subsection{Investigation of the damping performance of the real-time tuned dynamic} absorber

The frequency responses obtained by the real-time control experiment are shown in Fig. 13, where the disturbance frequency is predicted by the embedded variable resistance sensor. In the figure, the responses of the primary system and the dynamic absorber are expressed as the displacement transmissibility $T R_{1}$ and $T R_{2}$, with respect to the displacement of the disturbance input. The curve with a single resonant peak in Fig. 13(a) corresponds to the case where the absorber mass is rigidly fixed to the primary structure, whereas the other lines correspond to the responses elicited in the cases where the damper stiffness is fixed at the respective applied currents. The remaining bold solid lines represent the responses elicited in the case where the natural frequency of the absorber is adaptively tuned to the instantaneous frequency of the disturbance. The figure shows that the tunable absorber sustainably reduces the primary system response amplitude to a value that is as 
small as the minimum amplitude, within the variable range of 25.8 to $37.4 \mathrm{~Hz}$. The same can be said for the time histories of the primary system displacement $x_{1}$ shown in Fig. 14, where the embedded variable resistance sensor is adopted for detecting the disturbance frequency. Approximately identical control results can be obtained by the use of both internal and external sensors, where an increase of the amplitude near the resonance is constantly suppressed by the tuned absorber. The fact is also supported by the comparison of the time histories of the DC power control signal $V_{\text {ctrl }}$ shown in Fig. 15. The signals supplied by both sensors through a controller increase almost linearly within the variable frequency range, and they correspond to each other. These results demonstrate that the frequency-tuned dynamic absorber using an MRE and with a sensing function works effectively for the vibration control of structures, while keeping the mechanism simple and self-contained.

\section{Conclusions}

In this study, the dynamic property of the stiffness controllable elastomer, known as MRE, was experimentally evaluated. The dynamic property test showed that a certain optimal composition of materials within the mixture, such as a $40 \mathrm{vol} \%$ iron content in this studied case, led to the maximum stiffness property change. The MRE was then used in a dynamic vibration absorber in order to provide the natural frequency tunability and the selfsensing ability. The absorber was further applied to the vibration control of a one-DOF structure in conjunction with a real-time stiffness switching algorithm, where the tuned frequency value was determined with respect to the instantaneous disturbance frequency detected by a self-sensing function of an MRE. Furthermore, the sweeping sine wave excitation results of a target structure demonstrated that the improved damping 
performance could be obtained by the real time frequency-tuned dynamic absorbers in comparison to the passive-type absorbers. Without the use of an external sensor, the suggested MRE-based, tunable dynamic absorber with a sensing function enhances the damping performance for the vibration control of structures in a self-contained fashion. In such a process, only a simple alteration is required to the stiffness part of the conventional passive-type absorber. A further research should be conducted for the realization of a coexisting variable stiffness and electrical resistance properties within a single, molded MRE piece.

\section{References}

[1] Den Hartog JP (1956) Mechanical Vibrations. McGraw-Hill, New York

[2] Nagaya K, Kurusu A, Ikai S, Shitani Y (1999) Vibration control of a structure by using a tunable absorber and an optimal vibration absorber under auto-tuning control. J Sound Vib 228 (4):773-792

[3] Walsh PL, Lamancusa JS (1992) A variable stiffness vibration absorber for minimization of transient vibrations. J Sound Vib 158(2):195-211

[4] Nagarajaiah S, Sonmez E (2007) Structures with semiactive variable stiffness single/multiple tuned mass dampers. J Struct Eng 133(1):67-77

[5] Liu X, Feng X, Shi Y, Wang Y, Shuai Z (2013) Development of a semi-active electromagnetic vibration absorber and its experimental study. J Vib Acoust 135(5): 051015

[6] Lokander M, Stenberg B (2003) Improving the magnetorheological effect in isotropic magnetorheological rubber materials. Polym Test 22:677-680

[7] Gong XL, Zhang XZ, Zhang PQ (2005) Fabrication and characterization of isotropic magnetorheological elastomers. Polym Test 24:669-676

[8] Tian TF, Zhang XZ, Li WH, Alici G, Ding J (2013) Study of PDMS based magnetorheological elastomers. J Phys: Conference Series 412:012038.

[9] Stepanov GV, Abramchuk SS, Grishin DA, Nikitin LV, Kramarenko EY, Khokhlov AR (2007) Effect of a homogeneous magnetic field on the viscoelastic behavior of magnetic elastomers. Polym 48:488-495 
[10] Koo JH, Khan F, Jang DD, Jung HJ (2010) Dynamic characterization and modeling of magnetorheological elastomers under compressive loadings. Smart Mater Struct 19(11):117002

[11] Shiga T, Okada A, Kurauchi T (2003) Magnetroviscoelastic behavior of composite gels. J Appl Polym Sci 58(4):787-792

[12] Jolly MR, Carlson JD, Muñoz BC, Bullions TA (1996) The magnetoviscoelastic response of elastomer composites consisting of ferrous particles embedded in a polymer matrix. J Intell Mater Syst Struct 7:613-622

[13] Davis LC (1999) Model of magnetorheological elastomers. J of Appl Phys 85(6):3348-3351

[14] Zhou GY (2003) Shear properties of a magnetorheological elastomer. Smart Mater Struct 12:139-146

[15] Yang J, Gong X, Deng H, Qin L, Xuan S (2012) Investigation on the mechanism of damping behavior of magnetorheological elastomers. Smart Mater Struct 21:125015

[16] Deng HX, Gong XL (2007) Adaptive tuned vibration absorber based on magnetorheological elastomer. J Intell Mater Syst Struct 18:1205-1210

[17] Lerner AA, Cunefare KA (2008) Performance of MRE-based vibration absorbers. J Intell Mater Syst Struct 19:551-563

[18] Popp KM, Kroger M, Li W, Zhang X, Kosasih PB (2010) MRE properties under shear and squeeze modes and applications. J Intell Mater Syst Struct 21(15):1471-1477

[19] Salzano TB, Calder CA, Dehart DW (1992) Embedded-strain-sensor development for composite smart structures. Exp Mech 32(3):225-229

[20] Wang X, Gordaninejad F, Calgar M, Liu Y, Sutrisno J, Fuchs A (2009) Sensing behavior of magnetorheological elastomers. J Mech Design 131:091004

[21] Bica I (2009) Influence of the transverse magnetic field intensity upon the electric resistance of the magnetorheological elastomer containing graphite microparticles. Mater Matters 63:2230-2232

[22] Tian TF, Li WH, Deng YM (2011) Sensing capabilities of graphite based MR elastomers. Smart Mater Struct 20(2):025022

[23] Li W, Kostidis K, Zhang X, Zhou Y (2009) Development of a force sensor working with MR elastomers. Proc IEEE/ASME Int Conf Adv Intell Mech:233-238

[24] Carlson JD, Jolly MR (2000) MR fluid, form and elastomer devices. Mechatronics:555-569 
[25] Shimizu N, Yamazaki H (1993) Development of vibration insulator using a new material silicone gel. Trans JSME C 59(568):3717-3724

[26] Spanos P, Zeldin B (2000) Pitfalls of deterministic and random analysis of systems with hysteresis. J Eng Mech 126:1108-1110

[27] Torvik P, Bagley R (1984) On the appearance of the fractional derivative in the behavior of real materials. J Appl Mech 51:294-298

[28] http://www.inaba-rubber.co.jp/en/index.html (Accessed 16 April 2015) 


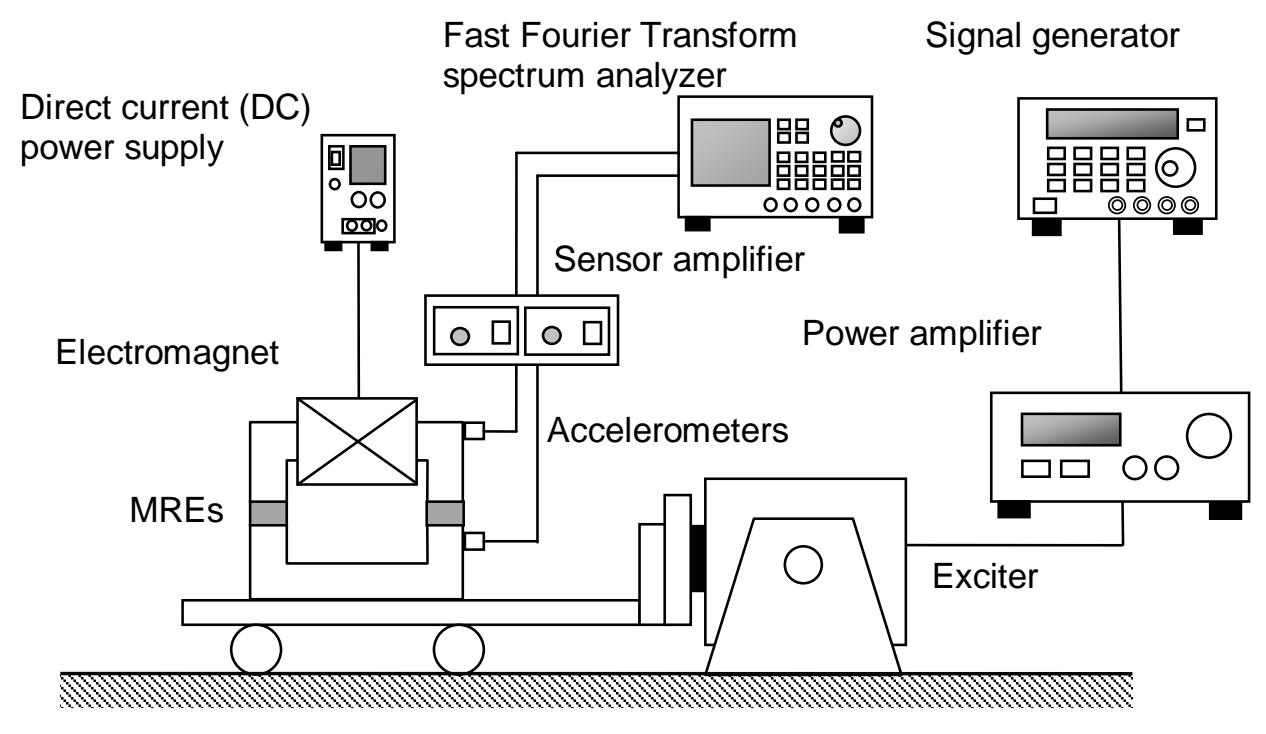

Fig. 1 Viscoelastic property measurement system 


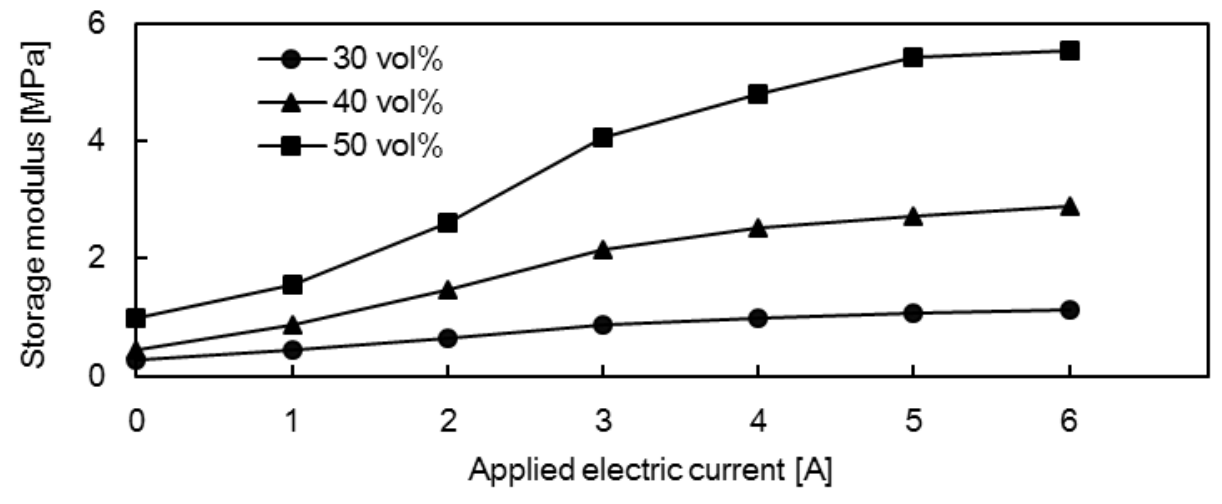

Fig. 2 Shear storage modulus of MREs plotted against the magnetic flux density 
(a)

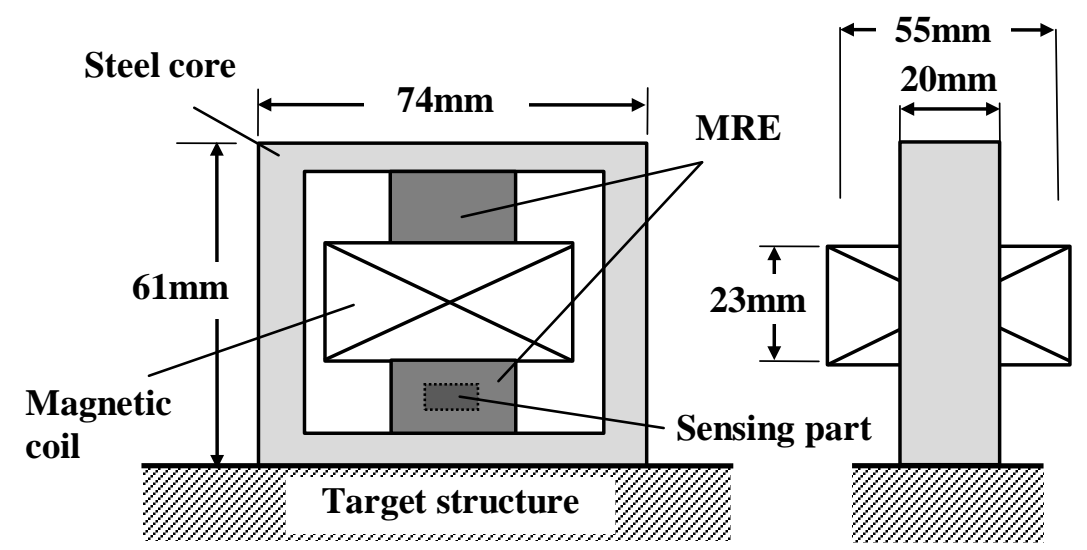

(b)

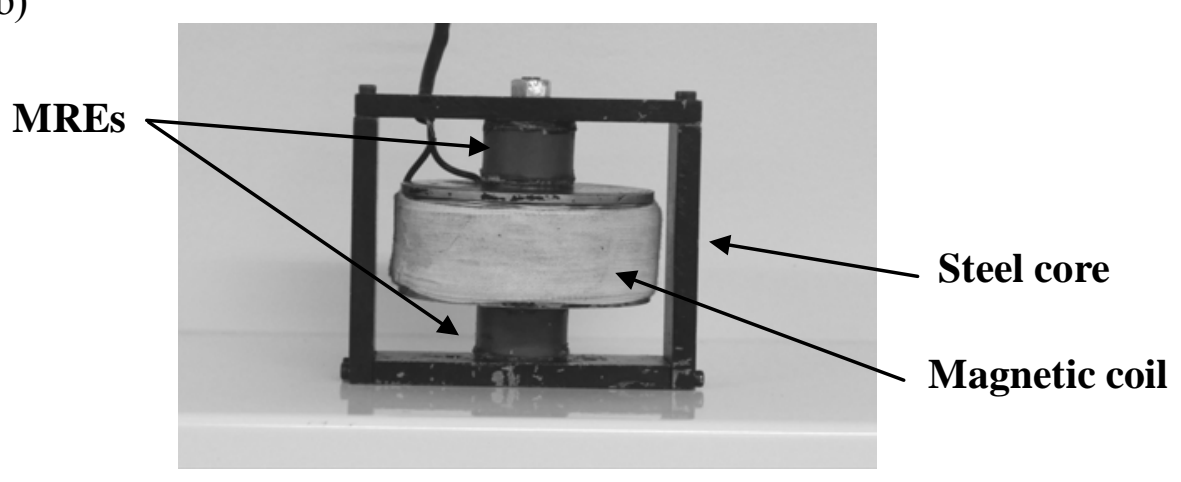

Fig. 3 A variable-stiffness dynamic absorber used in the present study: (a) schematic and (b) a photograph of the assembly 


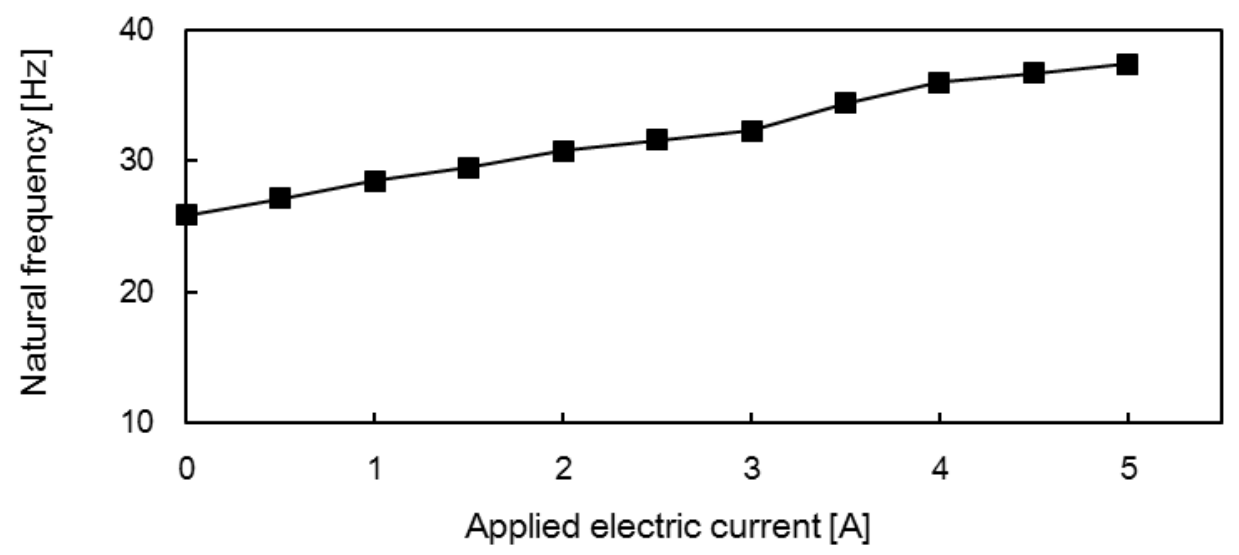

(a)

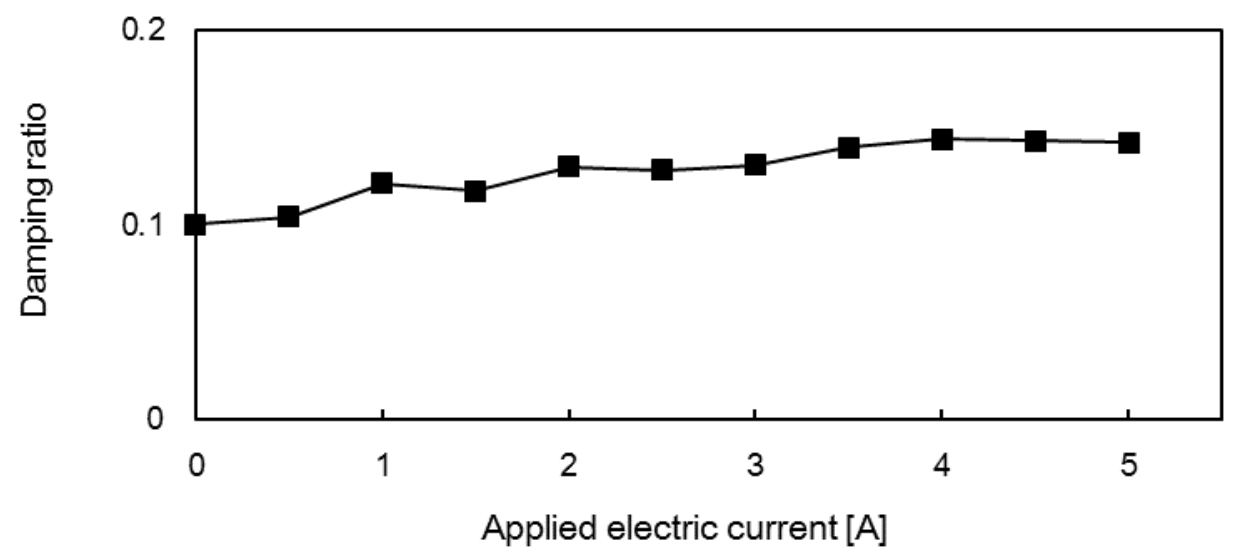

(b)

Fig.4 Fundamental properties of the variable-stiffness dynamic absorber: (a) natural frequency variation, and (b) damping ratio 
(a)

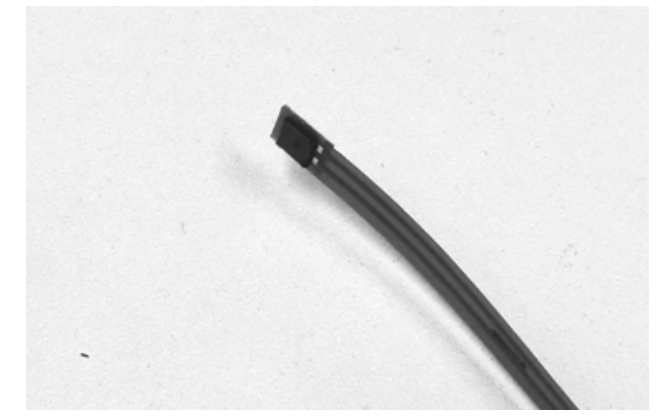

(b)

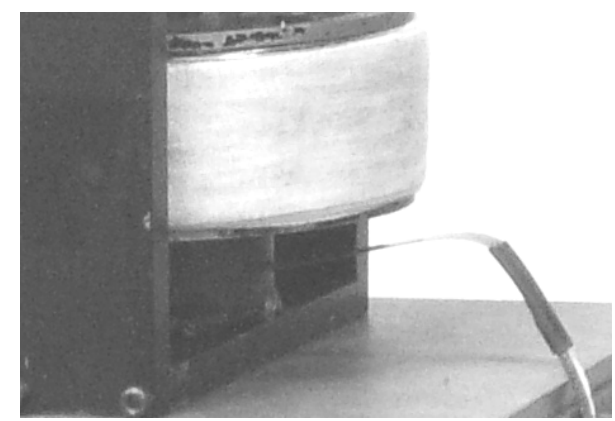

Fig. 5 A graphite-based elastomer used as a sensor. (a) An elastomer bonded with lead wire, and (b) the sensor embedded within a host MRE 


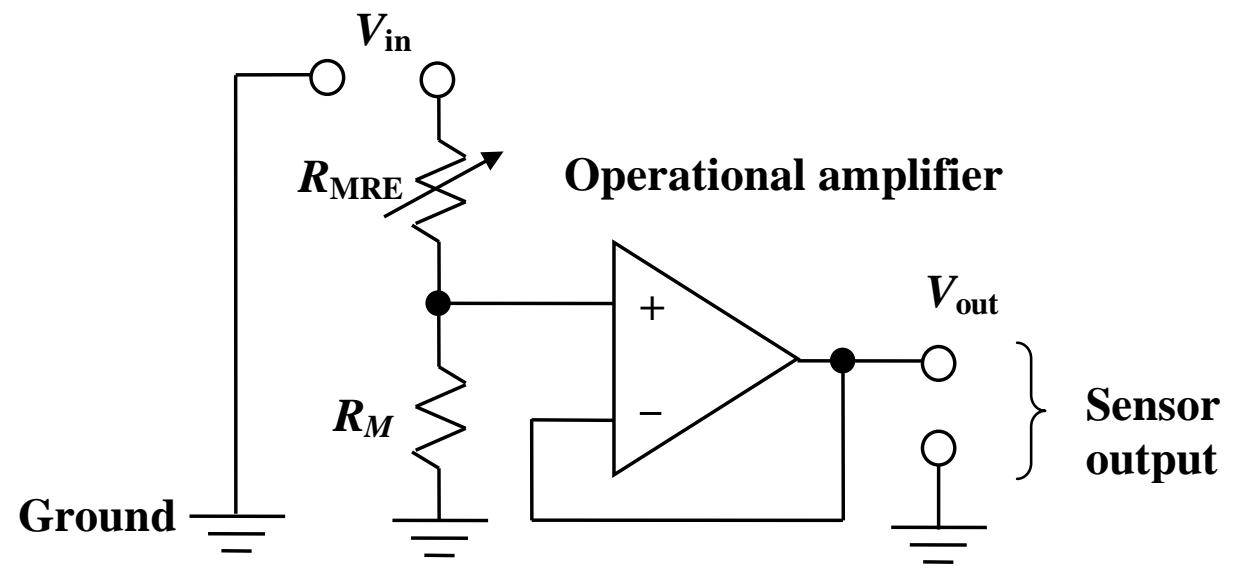

Fig. 6 A resistance voltage divider circuit used for stain-voltage conversion of MRE 


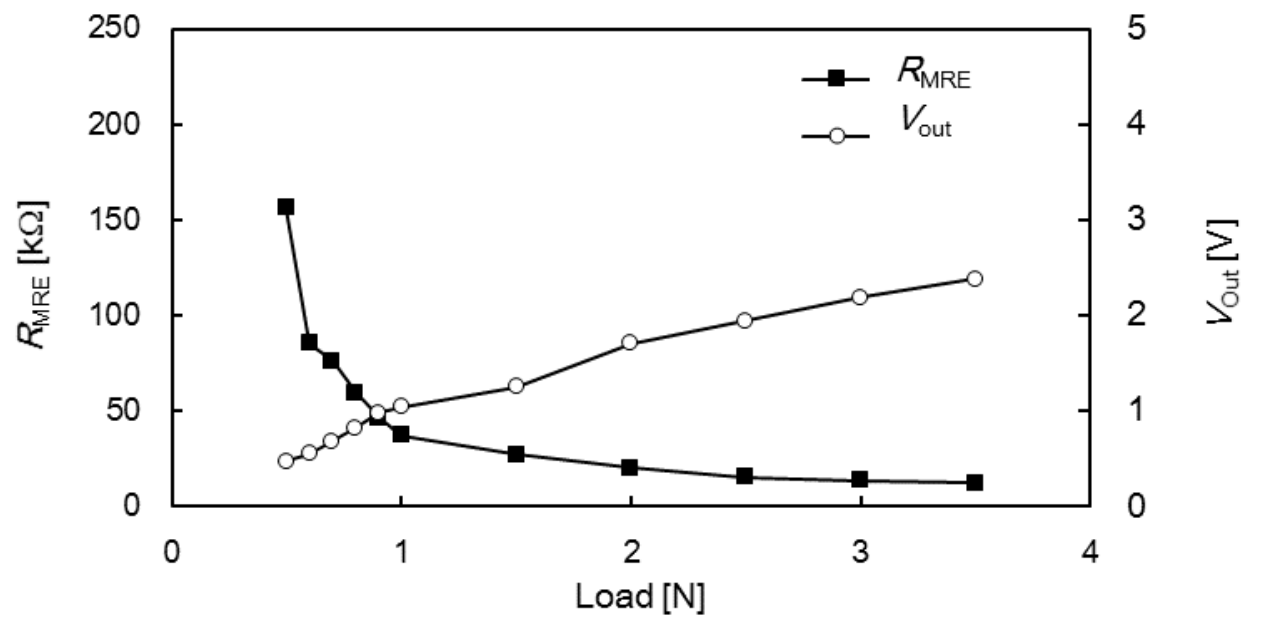

(a)

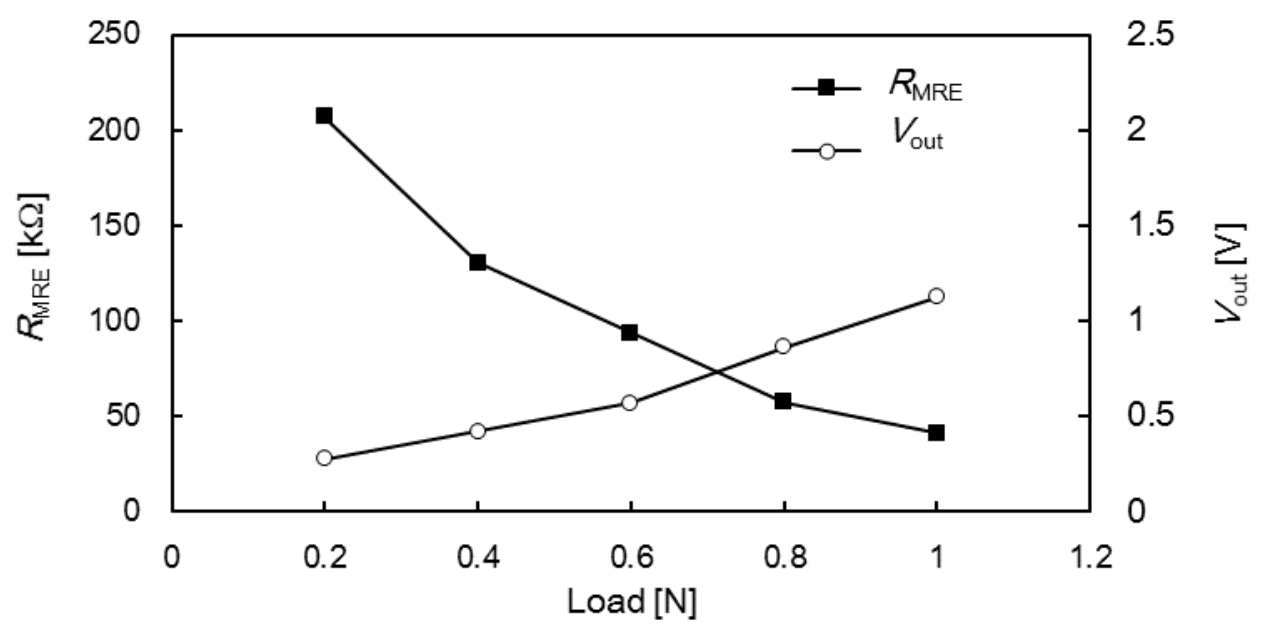

(b)

Fig. 7 The electrical resistance and the output voltage characteristics of a graphite-based elastomer. The characteristics against (a) compressive loadings, and (b) against shear loadings 


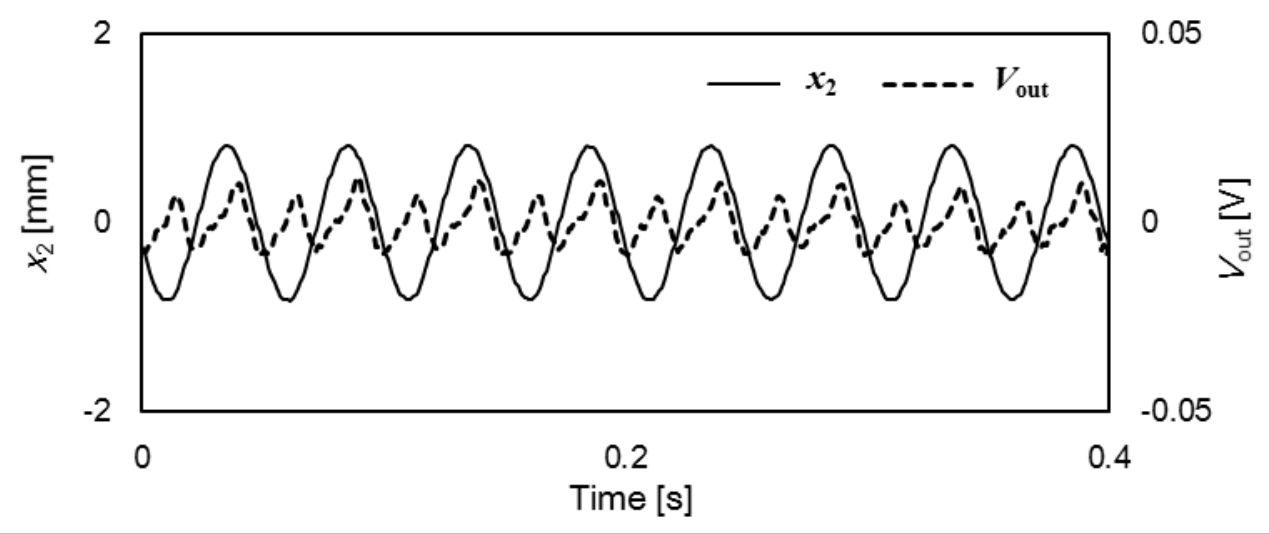

(a)

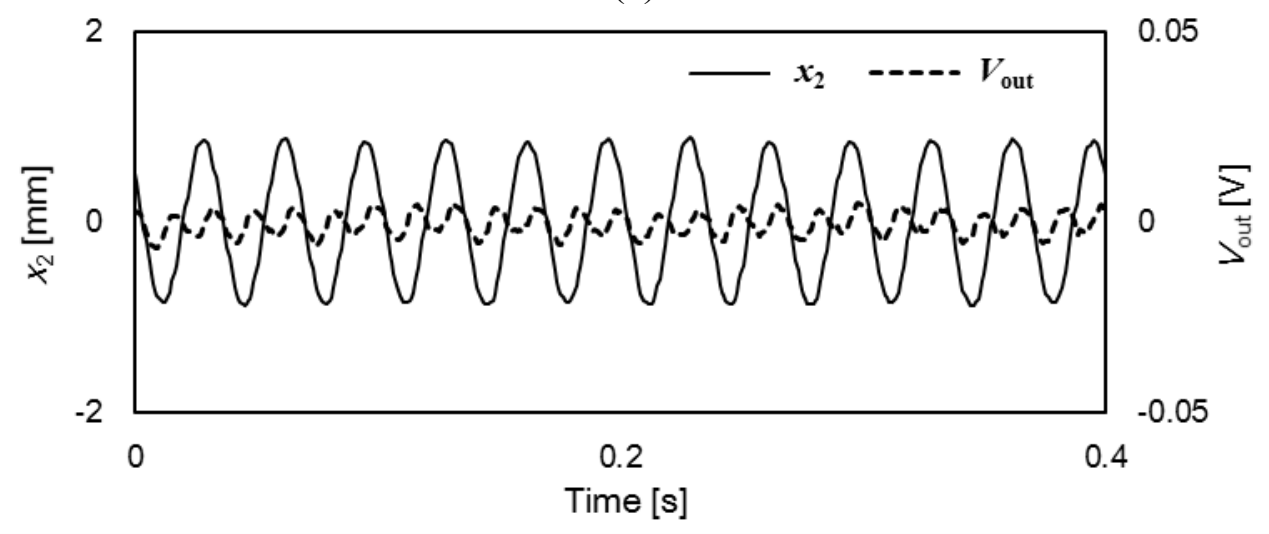

(b)

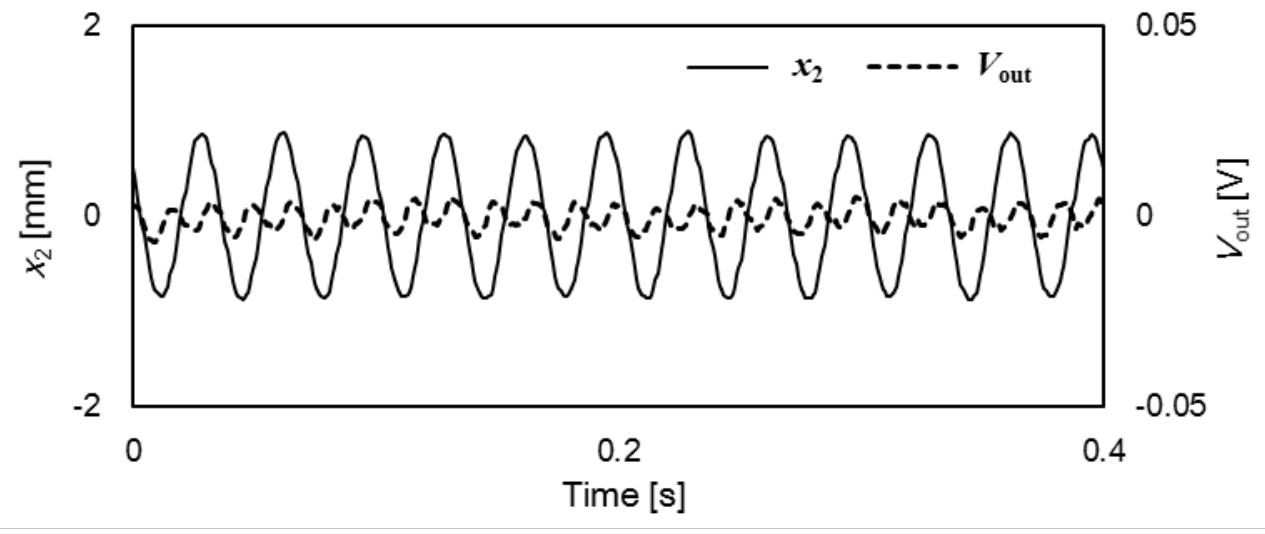

(c)

Fig. 8 Comparison of the time history of the variable resistance sensing function of the MRE and the displacement sensor at a (a) $20 \mathrm{~Hz}$ sinusoidal excitation, (b) $30 \mathrm{~Hz}$ excitation, and (c) $40 \mathrm{~Hz}$ excitation 


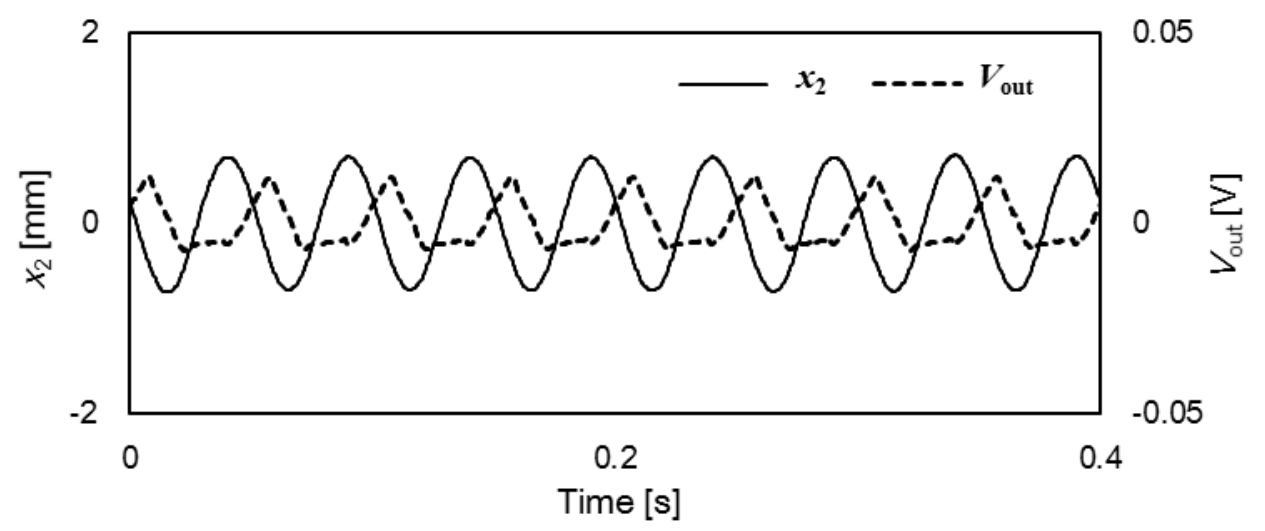

(a)

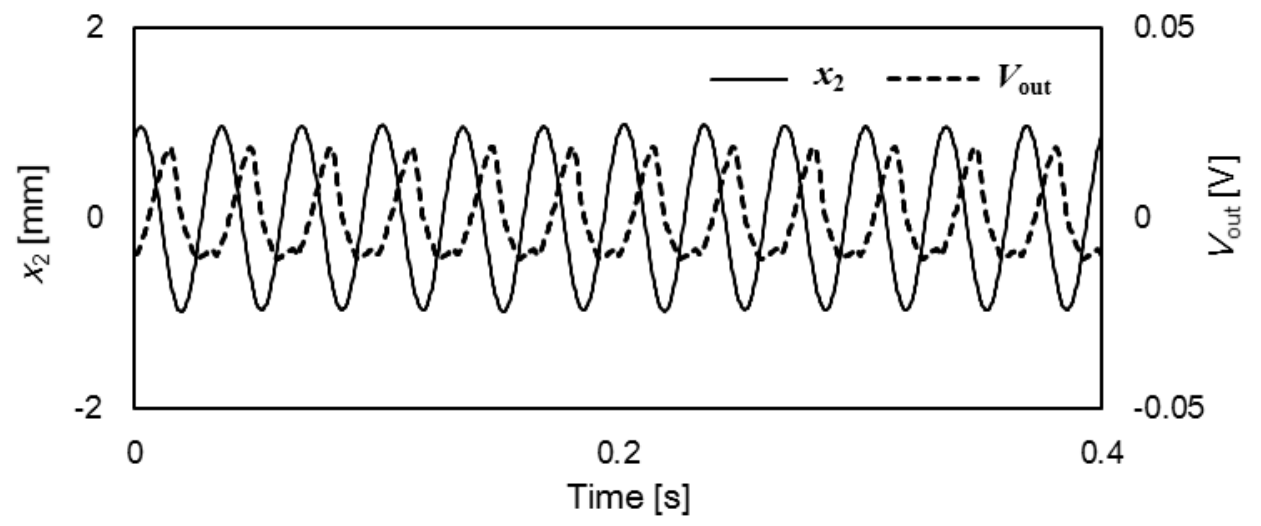

(b)

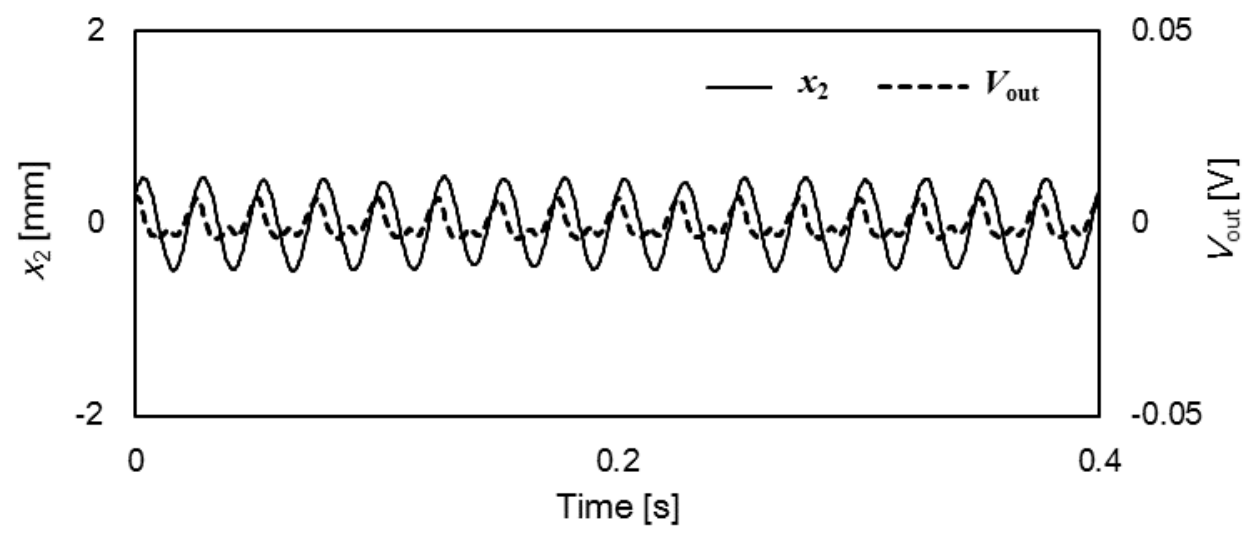

(c)

Fig. 9 Comparison of the time history of the sensor output of the biased MRE and the displacement sensor at a (a) $20 \mathrm{~Hz}$ sinusoidal excitation, (b) $30 \mathrm{~Hz}$ excitation, and (c) $40 \mathrm{~Hz}$ excitation 


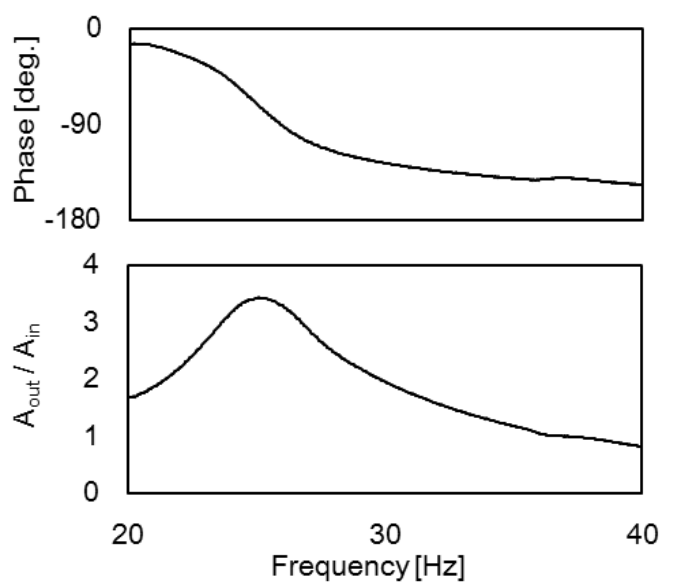

(a)

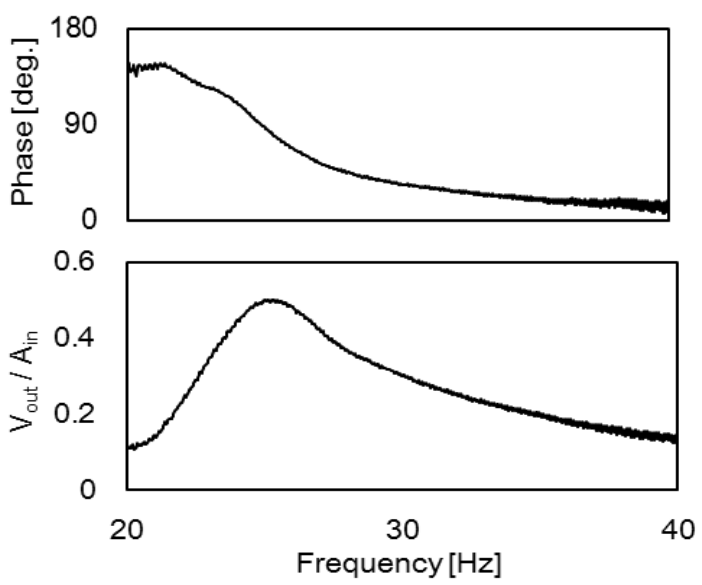

(b)

Fig. 10 The frequency responses of (a) the DVA calculated as the acceleration transfer function, and (b) the sensor output of a biased MRE 


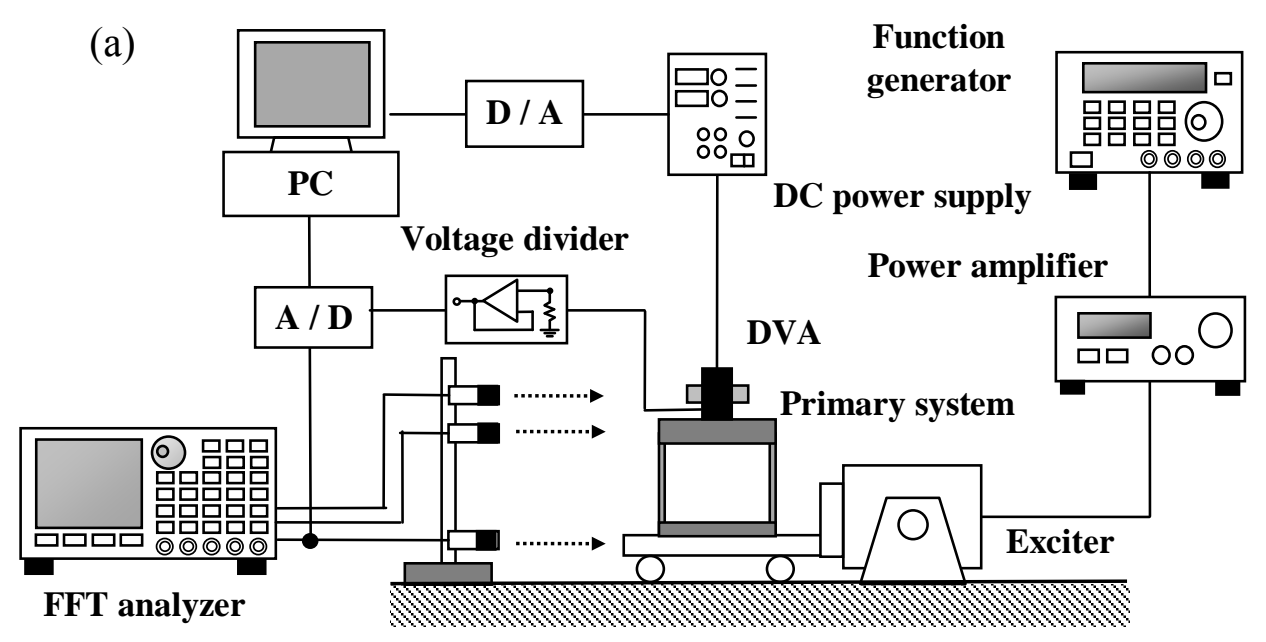

Displacement sensors

(b)

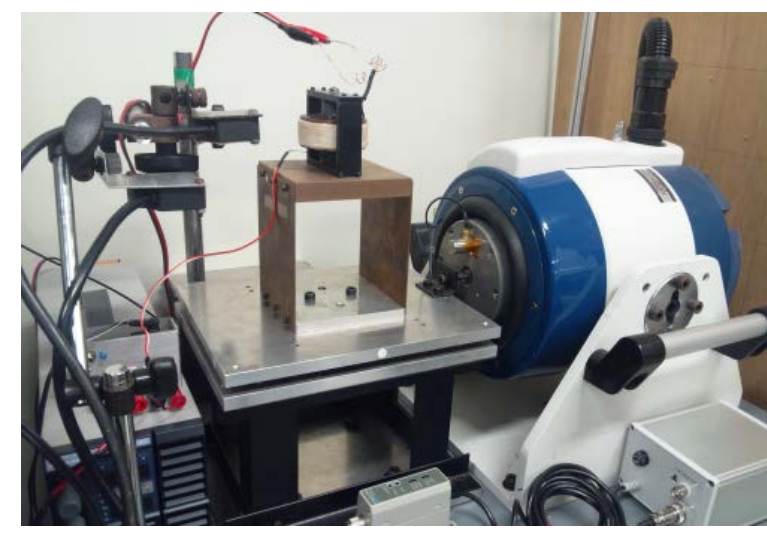

Fig. 11 Experimental setup of a base-excited primary system damped by a variable-stiffness DVA. (a) A schematic, and (b) a photograph of the assembly 


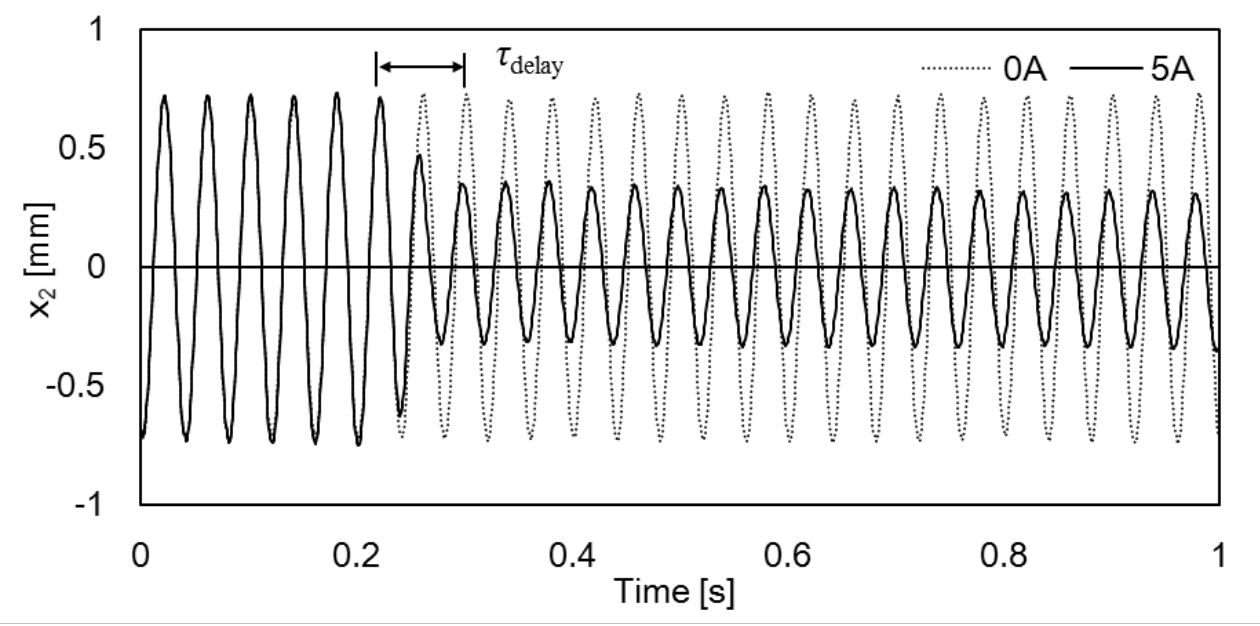

Fig. 12 Time history of the DVA displacement when the DVA is harmonically excited at 20 $\mathrm{Hz}$. Time delay occurs when switching the stiffness of MREs by applying an electric current of $5 \mathrm{~A}$ 


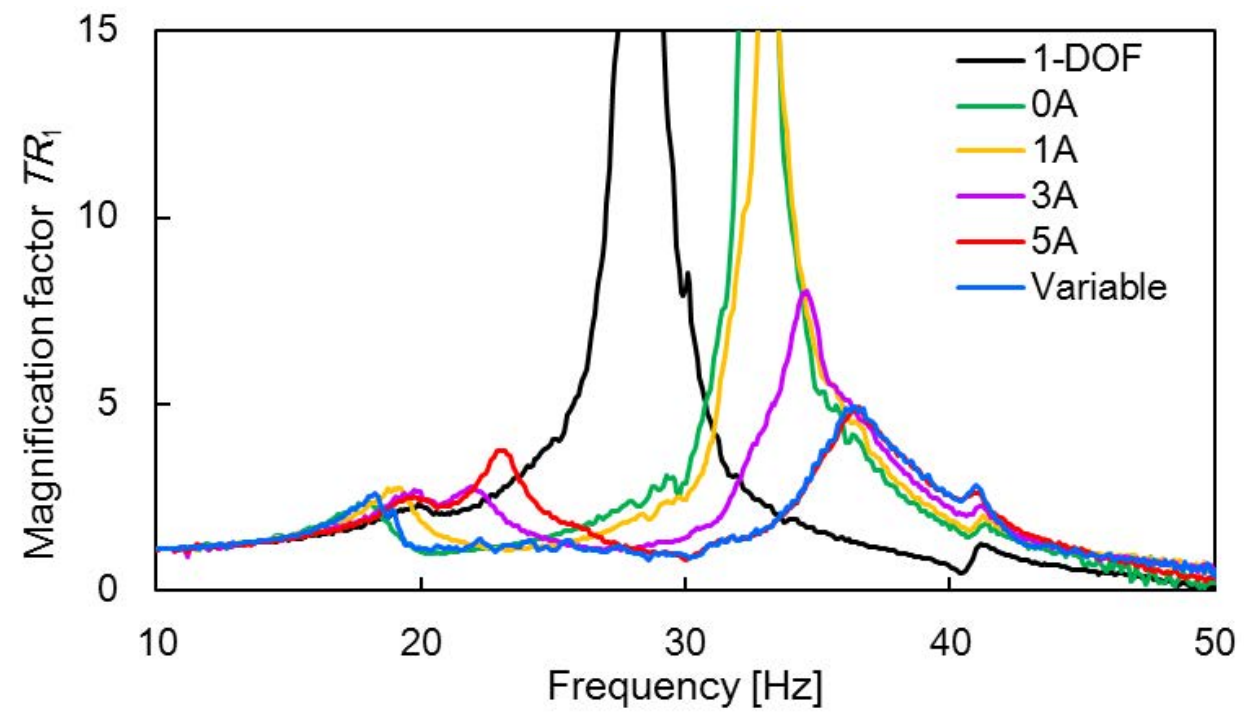

(a)

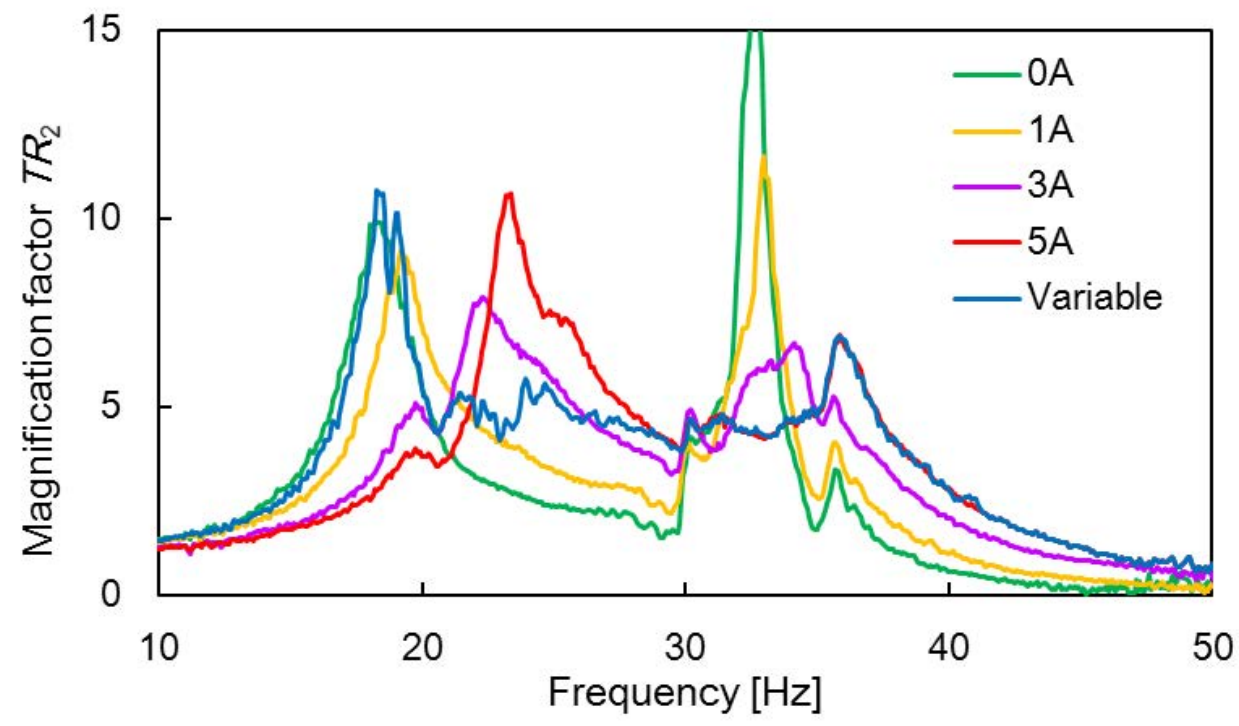

(b)

Fig. 13 Frequency response curves when the primary system is damped by DVA with an MRE with an iron concentration of $40 \%$. (a) The primary system responses, and (b) the DVA responses 


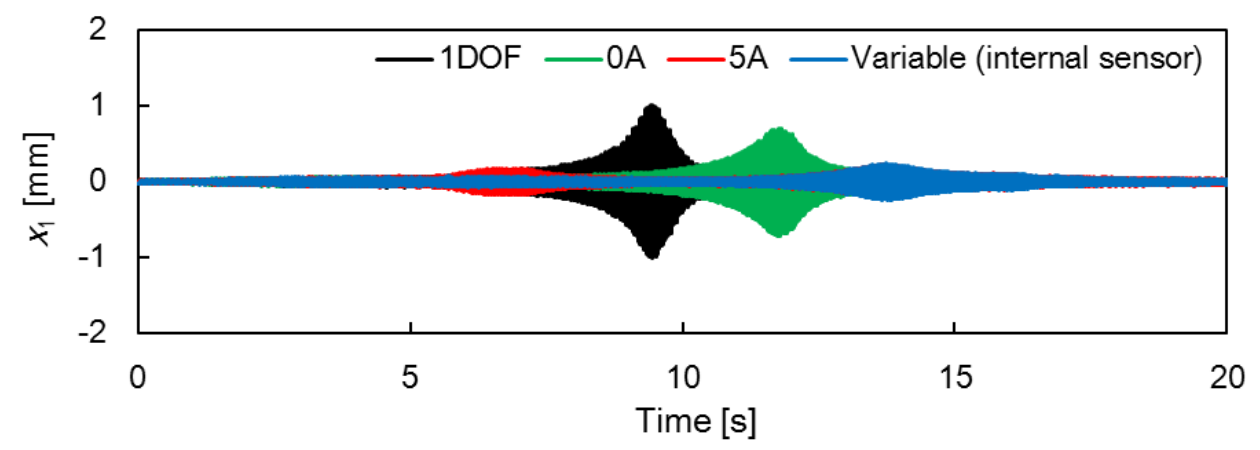

(a)

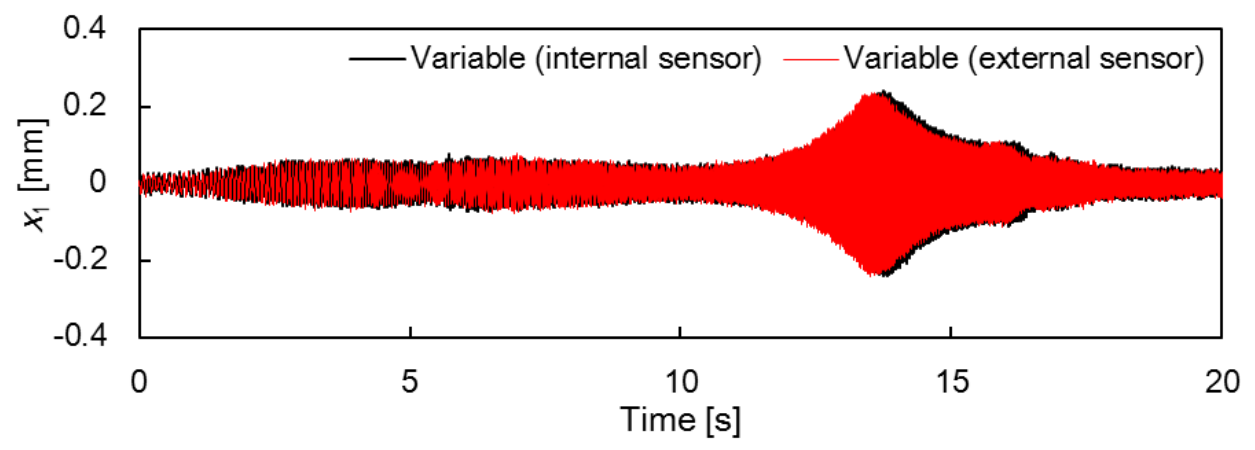

(b)

Fig. 14 Time histories of the primary system response elicited by sweeping a sine wave input at $2 \mathrm{~Hz} / \mathrm{s}$. In (a), the response with an adaptively tuned absorber is compared with property fixed cases, and in (b), the responses are compared for different sensors 


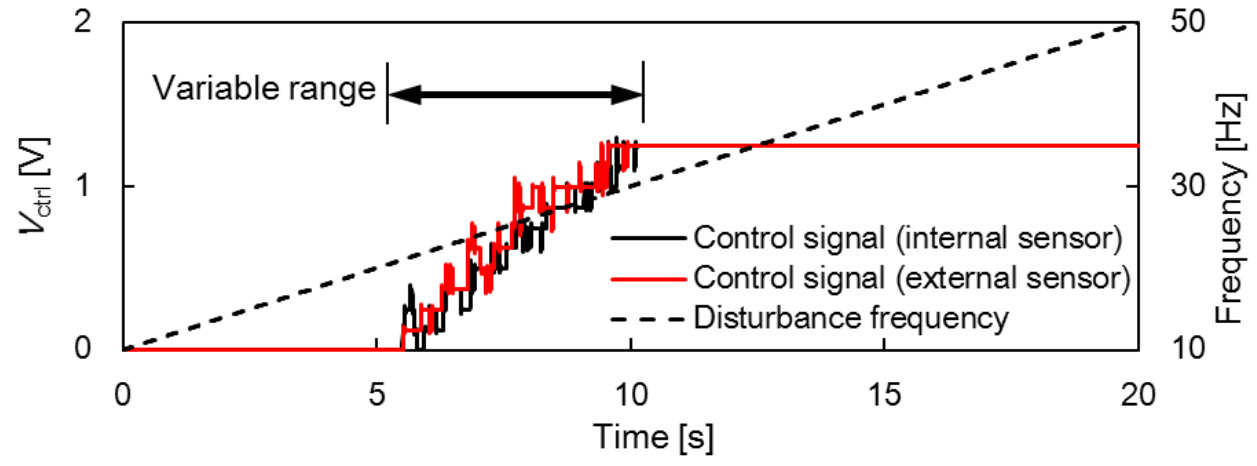

Fig. 15 DC power control signal supplied by the controller according to the detected instantaneous disturbance frequency 
Table 1 Average stiffness magnification and loss factor of MREs

\begin{tabular}{|c|c|c|c|c|c|c|}
\hline \multirow{2}{*}{$\begin{array}{c}\text { Current } \\
\text { (Magnetic flux density) }\end{array}$} & \multicolumn{2}{|c|}{30 vol\% } & \multicolumn{2}{|c|}{40 vol\% } & \multicolumn{2}{|c|}{50 vol\% } \\
\hline & $v$ & $\eta$ & $v$ & $\eta$ & $v$ & $\eta$ \\
\hline $0 \mathrm{~A}(0 \mathrm{mT})$ & 1.0 & 0.30 & 1.0 & 0.37 & 1.0 & 0.37 \\
\hline $1 \mathrm{~A}(59 \mathrm{mT})$ & 1.7 & 0.36 & 2.2 & 0.43 & 1.7 & 0.40 \\
\hline $2 \mathrm{~A}(113 \mathrm{mT})$ & 2.5 & 0.40 & 3.7 & 0.46 & 2.8 & 0.41 \\
\hline $3 \mathrm{~A}(167 \mathrm{mT})$ & 3.4 & 0.41 & 5.5 & 0.46 & 4.4 & 0.40 \\
\hline $4 \mathrm{~A}(218 \mathrm{mT})$ & 3.9 & 0.40 & 6.4 & 0.46 & 5.2 & 0.44 \\
\hline $5 \mathrm{~A}(267 \mathrm{mT})$ & 4.3 & 0.40 & 6.9 & 0.43 & 5.8 & 0.40 \\
\hline $6 \mathrm{~A}(316 \mathrm{mT})$ & 4.5 & 0.41 & 7.4 & 0.44 & 6.0 & 0.39 \\
\hline
\end{tabular}


Table 2 Variation characteristic of the variable-stiffness dynamic absorber

\begin{tabular}{ccccc}
\hline $\begin{array}{c}\text { Iron } \\
\text { concentration }\end{array}$ & $\begin{array}{c}\text { Frequency } \\
\text { variation range }\end{array}$ & Bandwidth & $\begin{array}{c}\text { Magnification to } \\
\text { baseline }\end{array}$ & Damping ratio \\
\hline $40 \mathrm{vol} \%$ & $25.8-37.4[\mathrm{~Hz}]$ & $11.6[\mathrm{~Hz}]$ & 1.45 & 0.12 \\
\hline
\end{tabular}

